\title{
K-Te photocathodes: A new electron source for photoinjectors
}

\author{
D. Bisero and B. M. van Oerle \\ NCLR, Post Office Box 2662, 7500 CR Enschede, The Netherlands
}

\author{
G. J. Ernst, J. W. J. Verschuur, and W. J. Witteman \\ University of Twente, Department of Applied Physics, Post Office Box 217, 7500 AE Enschede, \\ The Netherlands
}

(Received 18 November 1996; accepted for publication 29 April 1997)

\begin{abstract}
$\mathrm{K}$-Te photocathodes deposited on a Mo substrate have been successfully used as an electron source in the free electron laser of University of Twente. Long lifetimes have been measured: after more than $20 \mathrm{~h}$ of operation in the accelerator a $\mathrm{K}-\mathrm{Te}$ cathode with $4.75 \%$ initial quantum efficiency still displays a $1.1 \%$ quantum efficiency at $259 \mathrm{~nm}$. Moreover, the quantum efficiency of this cathode versus operation time can be fitted by an exponential decay curve, which saturates asymptotically to a $1.03 \%$ value, suggesting that a quantum efficiency close to $1 \%$ could be sustained for very long operation times. Films degraded by use can be recovered to a quantum efficiency which is close to the initial value, by heating the substrate at temperatures between 100 and $330{ }^{\circ} \mathrm{C}$. A new procedure to obtain K-Te cathodes with high (up to 11\%) quantum efficiencies is described. (C) 1997 American Institute of Physics. [S0021-8979(97)05715-0]
\end{abstract}

\section{INTRODUCTION}

Photocathodes irradiated by a pulsed laser can produce very bright electron beams, with very high current densities. ${ }^{1}$ For this reason, in past years, the electron accelerator and free electron laser (FEL) communities have been searching for a suitable material to be used as a photocathode in photoinjectors. ${ }^{2-4}$ High $(>1 \%)$ quantum efficiency (QE) for photoemission by visible or soft UV light and long lifetime under operating conditions are desired photocathode's features, difficult to be matched. Recently, we have demonstrated the photoemissive properties of $\mathrm{K}-\mathrm{Te}$ semiconductor films evaporated under ultrahigh vacuum (UHV) conditions. ${ }^{5}$ Their high QE at $259 \mathrm{~nm}$ and long lifetime after storage in UHV conditions, have suggested the possibility of using $\mathrm{K}-\mathrm{Te}$ films as photocathodes in electron accelerators. We have verified this possibility, operating the electron accelerator of the Twente FEL with K-Te photocathodes and investigating both their lifetime under operating conditions and their regeneration by substrate heating.

\section{EXPERIMENT}

The $\mathrm{K}-\mathrm{Te}$ photocathodes have been evaporated in the preparation chamber of the Twente FEL, ${ }^{6}$ on a Mo substrate. Details concerning the preparation procedure can be found elsewhere. ${ }^{5}$ A mercury lamp with different bandpass interference filters has been used to illuminate the photocathode (biased by $-90 \mathrm{~V}$ ) with light of different wavelengths, in order to measure the photocurrent with a picoammeter. The optimized Te evaporation time already determined in previous investigations ${ }^{5}$ has been used for the preparation of all the cathodes reported in this article.

The electron beam has been generated in a $1.3 \mathrm{GHz}$ if linac operating at a nominal energy of $6 \mathrm{MeV}$. In this system, the drive laser $^{7}$ used to illuminate the photocathode is a mode-locked Nd:YLF laser delivering $60 \mathrm{ps}$ pulses at a repetition rate of $81.25 \mathrm{MHz}$ and at a wavelength of $1053 \mathrm{~nm}$. These pulses are compressed in a fiber-grating pulse com- pressor to a duration of $20 \mathrm{ps}^{8}{ }^{8}$ Then, a pulse train of $10 \mu \mathrm{s}$ duration, containing 800 pulses, is sliced out by a traveling wave acousto-optic modulator. This pulse train is amplified in two double pass flashlamp pumped Nd:YLF amplifiers. Since the K-Te cathodes are sensitive in the UV spectral range, the pulse train is frequency quadrupled in order to obtain a wavelength of $263 \mathrm{~nm} .{ }^{9}$ Finally, the K-Te photocathodes are illuminated by trains of 10-ps long UV pulses, with energy between 1 and $2 \mu \mathrm{J}$. The electron beam produced in this way for the lifetime experiments had a charge per bunch between 1 and $4 \mathrm{nC}$ at an electron energy of 6 $\mathrm{MeV}$.

\section{RESULTS AND DISCUSSION}

\section{A. Lifetime}

In order to investigate the lifetime of the $\mathrm{K}-\mathrm{Te}$ cathodes, QE measurements were performed as a function of operating time. To this purpose, the accelerator operation was stopped for a few minutes and the photocathodes were moved under UHV conditions to the preparation chamber, where the photocurrent produced by the $\mathrm{Hg}$ lamp was measured. The QE measured with this procedure is estimated to be a factor of three higher than the $\mathrm{QE}$ obtained under operating conditions by measuring the electron beam current and the drive laser power. Every operating session lasted on the average $3 \mathrm{~h}$; during the time between successive sessions, the photocathodes were kept in the preparation chamber, at a pressure in the low $10^{-10}$ Torr range. During operation, the maximum pressure in the accelerator was $5 \times 10^{-9}$ Torr. The cathodes have been used in these experiments until their QE dropped to a value lower than half the initial one. Figure 1 shows the $\mathrm{QE}$ as a function of operation time for the $\mathrm{K}-\mathrm{Te}$ cathodes, evaporated at three different substrate temperatures $(26,90$, and $120^{\circ} \mathrm{C}$ ). From this figure, the half-life of the cathodes has been determined and reported in Table I. The same definition used in Ref. 5 for different preparation procedures has been reported here. In fact, it was shown that different be- 


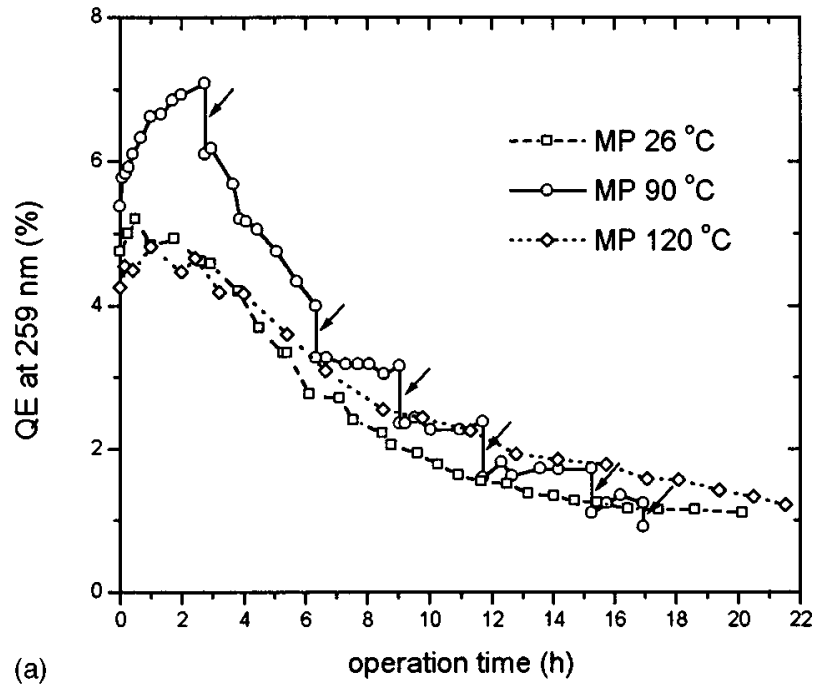

(a)

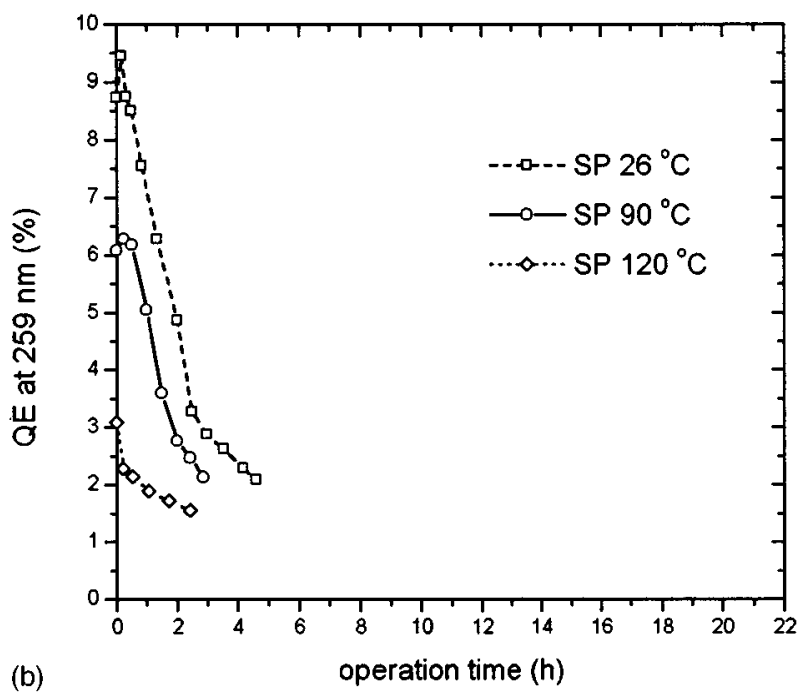

FIG. 1. QE at $259 \mathrm{~nm}$ as a function of operation time for K-Te MP cathodes (a) and SP cathodes (b) evaporated at substrate temperatures of 26, 90, and $120^{\circ} \mathrm{C}$.

haviors of the $\mathrm{K}-\mathrm{Te}$ films can be obtained, once the Te evaporation has been accomplished, either by stopping the $\mathrm{K}$ evaporation when the $\mathrm{QE}$ is at its maximum (procedure named MP) or by continuing $\mathrm{K}$ evaporation after the QE maximum (procedure named SP). In the latter case, a steep decrease of the QE occurs after the peak, followed by a smooth increase which finally saturates to a value that is always lower than the maximum one. It must be noticed that

TABLE I. Time necessary to reduce the $\mathrm{QE}$ at $259 \mathrm{~nm}$ to half the initial value (half-life) of the $\mathrm{K}-\mathrm{Te}$ photocathodes.

\begin{tabular}{rc}
\hline \hline $\mathrm{K}-\mathrm{Te}$ cathode & Half-life (h) \\
\hline MP $120^{\circ} \mathrm{C}$ & 11.9 \\
MP $90^{\circ} \mathrm{C}$ & 9 \\
MP $26{ }^{\circ} \mathrm{C}$ & 7.7 \\
SP $120^{\circ} \mathrm{C}$ & 2.4 \\
SP $90^{\circ} \mathrm{C}$ & 1.83 \\
SP $26^{\circ} \mathrm{C}$ & 2.2 \\
\hline \hline
\end{tabular}

after a MP evaporation the QE of a $\mathrm{K}$-Te cathode undergoes an exponential decay, with a characteristic time of the order of minutes, after which a stable QE value is reached. In the lifetime experiments involving the MP cathodes reported in this article, the initial QE refers to the stable value reached a few hours after the evaporation process has been accomplished.

The half-life values reported in Table I clearly show how the type of procedure (MP or SP) strongly affects the lifetime of the K-Te films. Photocathodes evaporated at a certain substrate temperature with a MP procedure have much longer half-lives than photocathodes evaporated at the same temperature with a SP procedure. We believe that this result is in agreement with the hypothesis ${ }^{5}$ that during the additional K evaporation time necessary to go from a MP to a SP $\mathrm{K}-\mathrm{Te}$ film the layer's chemical composition changes (for example from $\mathrm{K}_{2} \mathrm{Te}_{3}$ to $\mathrm{K}_{2} \mathrm{Te}_{2}$ ).

Long $\mathrm{K}-\mathrm{Te}$ lifetimes under operating conditions have been observed for all the MP cathodes. Here we are not referring to the half-life, but to an operative definition of lifetime, which has more to do with "usability" in the FEL and with the concept of "high" QE already stated in the introduction and used by other authors. ${ }^{4}$ Therefore, what we will consider as lifetime in the following discussion is the operation time after which the QE drops to a value close to $1 \%$. This time was determined in this article only for MP cathodes, since they are the ones showing the best performances. Let us analyze in detail the behavior of these cathodes.

MP $120^{\circ} \mathrm{C}$ : after more than $21 \mathrm{~h}$ of operation, this cathode displays a $\mathrm{QE}$ of $1.22 \%$. The degradation process occurs during operation and no change of the QE has been detected after storage in the preparation chamber between successive operations. The dependence of the QE on the operation time appears to become inversely proportional for long times.

MP $90{ }^{\circ} \mathrm{C}$ : after about $17 \mathrm{~h}$ of operation, this cathode has a $1.24 \% \mathrm{QE}$, which drops below $1 \%$ during storage in the preparation chamber. Figure 1(a) shows that during the first $3 \mathrm{~h}$ of operation the QE increases; then, during the storage in the preparation chamber after operation, the QE decreases. This effect occurs every time operation is stopped, as indicated by arrows in the figure, drawn where two points have been reported in correspondence of the same time value (the highest is the $\mathrm{QE}$ at the end of operation, the lowest the $\mathrm{QE}$ after about $20 \mathrm{~h}$ of storage in the preparation chamber after an operation session and before the following one). The only significant degradation of the cathode's QE during operation occurs between about the 3rd and the 6th hour, after which a step structure in the QE dependence on operation time can be clearly seen, corresponding to a nearly constant QE during operation and a decrease of $\mathrm{QE}$ taking place during storage after operation. This behavior suggests that longer lifetimes could have been achieved with this cathode if operation sessions were longer.

MP $26{ }^{\circ} \mathrm{C}$ : after more than $20 \mathrm{~h}$ of operation, this cathode displays a QE of $1.1 \%$. The degradation process occurs during operation, whereas during storage in the preparation chamber between successive operations a small increase of the QE [of the order of $0.1 \%-0.2 \%$ and not reported in Fig. 


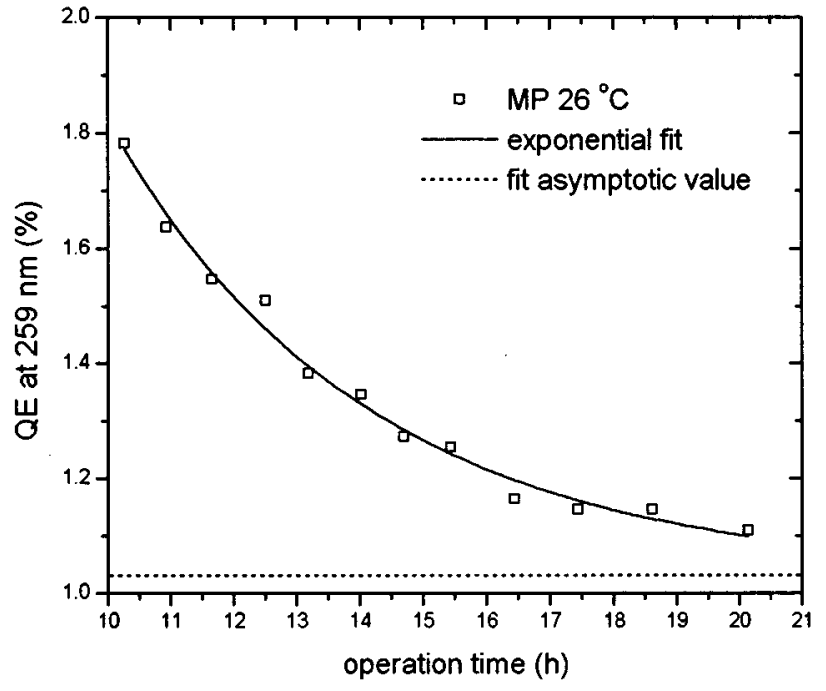

FIG. 2. QE at $259 \mathrm{~nm}$ as a function of operation time $(t>10 \mathrm{~h})$ of a MP $26{ }^{\circ} \mathrm{C}$ cathode. The exponential best fit (continuous line representing the function $f(t)=1.03147+0.7397 e^{-(t-10.26667) / 4.12997}$ ) and its asymptot (dotted line) are reported.

$1(\mathrm{a})$ ] is detected. Since the dependence of the QE of this cathode on operation time seems to follow an exponential decay for long times, the best fit of the last part of the curve (from about $10 \mathrm{~h}$ to the end) is superimposed on the experimental data in Fig. 2. From the analytical expression of the fit, an asymptotic $1.03147 \%$ value of the QE, reached around $t=60 \mathrm{~h}$, can be immediately inferred, suggesting that this cathode can be expected to hold for a very long time, probably much longer than the operation time used in this experiment, with a $\mathrm{QE}$ value very close to $1 \%$. It must be noticed that the average QE of the MP $26{ }^{\circ} \mathrm{C}$ cathodes evaporated so far in our preparation chamber $(6.5 \%)$ is definitely higher than the initial QE of the cathode used in the lifetime experiment $(4.75 \%)$. This circumstance makes us confident that higher saturation QE values could be obtained with this kind of $\mathrm{K}$-Te photocathode.

From the analysis of the degradation during accelerator operation of the MP cathodes evaporated at different temperatures, some conclusions can be drawn. The lifetime does not appear to depend significantly on the substrate temperature during evaporation: the MP cathodes $\mathrm{QE}$ drops to a value close to $1 \%$ in a time that is about $20 \mathrm{~h}$ for all of them. What seems to be clearly different is the shape of the QE decrease with operation time for long times (say more than $10 \mathrm{~h}$ of operation), that is linear for the MP cathode evaporated at $120^{\circ} \mathrm{C}$, step shaped for the MP $90{ }^{\circ} \mathrm{C}$ cathode and exponential for the MP $26^{\circ} \mathrm{C}$ cathode. We believe that this circumstance could make the difference for operation times much longer than the ones of the described experiment. To this respect, the MP $26^{\circ} \mathrm{C}$ cathode could display the best performances, due to the observed QE saturation effect. Nevertheless, it must be underlined that a correlation between shape of the QE versus operation time and substrate temperature during evaporation can not be assessed on the basis of the presented data and needs further investigations to be established.

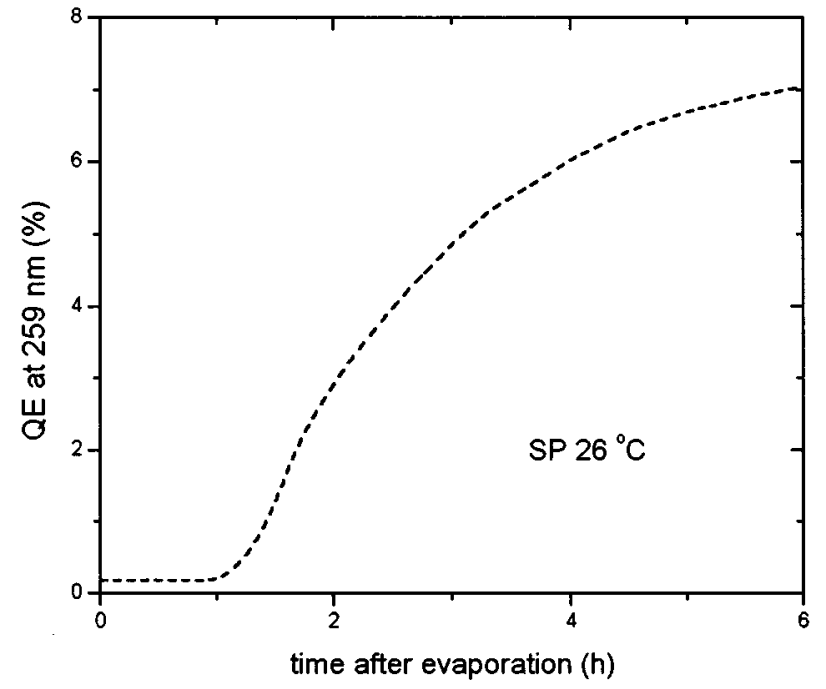

FIG. 3. Increase of the QE at $259 \mathrm{~nm}$ after evaporation of a SP $26^{\circ} \mathrm{C}$ cathode.

It is worth noticing that Fig. 1(b) also includes data concerning a $26{ }^{\circ} \mathrm{C}$ SP cathode, which start from an $8.73 \%$ initial QE. This fact is not in contradiction with a previously reported result, ${ }^{5}$ demonstrating that a SP photocathode evaporated at the substrate temperature of $26{ }^{\circ} \mathrm{C}$ shows a very low $\mathrm{QE}$ value (about $0.1 \%$ ). In Ref. 5, it was tentatively ascribed to the formation of a $\mathrm{K}$ layer at the surface of the cathode, due to inefficient diffusion at room temperature of $\mathrm{K}$ through the already formed $\mathrm{K}-\mathrm{Te}$ layer. In fact, expecting this $\mathrm{K}$ excess could be unstable, we have measured the QE of a SP $\mathrm{K}-\mathrm{Te}$ cathode evaporated at $26{ }^{\circ} \mathrm{C}(\mathrm{QE}$ maximum during evaporation $9.86 \%$, saturation $\mathrm{QE}$ value at the end of evaporation $0.18 \%$ ) for very long times after evaporation. During the first hour, the QE was constant, but then it started to increase slowly and after about $20 \mathrm{~h}$ a $9 \% \mathrm{QE}$ value was reached. The first $6 \mathrm{~h}$ of the $\mathrm{QE}$ increase after evaporation

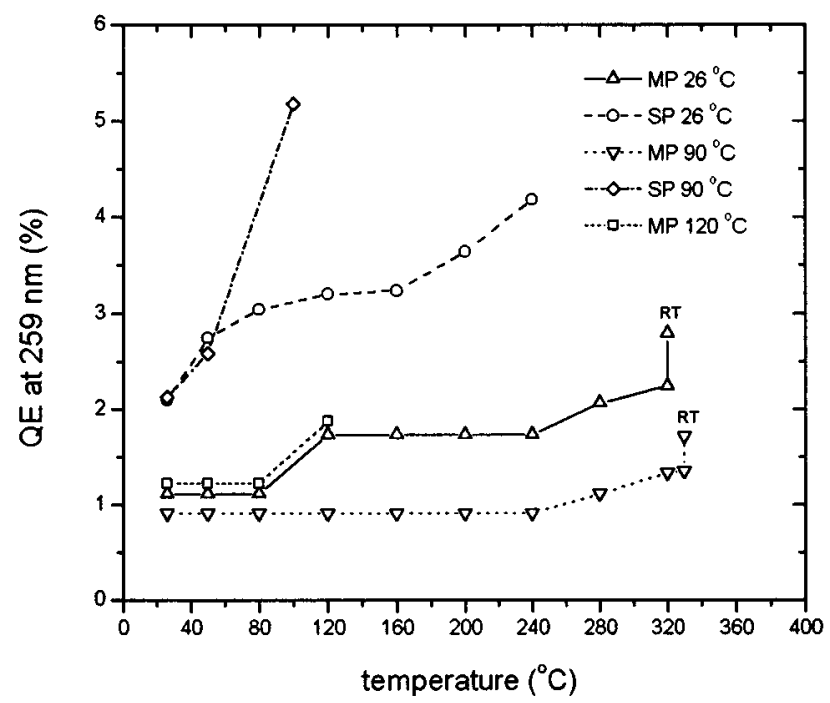

FIG. 4. Regeneration by heating of the K-Te cathodes. The stable QE value reached after the photocathode has been kept at a constant temperature in the range of $50-330^{\circ} \mathrm{C}$ for about $20 \mathrm{~min}$ is reported. 
TABLE II. Regeneration of the K-Te photocathodes: the ratio between the $\mathrm{QE}$ after regeneration by heating and the QE after evaporation has been reported.

\begin{tabular}{cc}
\hline \hline K-Te cathode & $\begin{array}{c}\text { Ratio between } \\
\text { recovered and initial QE }\end{array}$ \\
MP $120^{\circ} \mathrm{C}$ & 0.44 \\
MP $90{ }^{\circ} \mathrm{C}$ & 0.32 \\
MP $26{ }^{\circ} \mathrm{C}$ & 0.59 \\
$\mathrm{SP} \quad 90^{\circ} \mathrm{C}$ & 0.85 \\
$\mathrm{SP} 26{ }^{\circ} \mathrm{C}$ & 0.48 \\
\hline \hline
\end{tabular}

are reported in Fig. 3. We believe there are two possible explanations of this result, both consistent with the formation of a $\mathrm{K}$ excess during evaporation:

(1) the $\mathrm{K}$ excess slowly diffuses through the already formed $\mathrm{K}-\mathrm{Te}$ layer and reacts with $\mathrm{Te}$;

(2) the K excess slowly unbinds and is lost; the observed QE is then due to the $\mathrm{K}$-Te layer evaporated before the $\mathrm{K}$ excess formation.

Other SP $26{ }^{\circ} \mathrm{C} \mathrm{K}$-Te cathodes have been evaporated in our preparation chamber after this experiment: the best QE obtained about $20 \mathrm{~h}$ after evaporation was $11 \%$, which is to our knowledge the highest stable QE at $259 \mathrm{~nm}$ ever reported for $\mathrm{K}-\mathrm{Te}$.

\section{B. Regeneration}

Degraded K-Te cathodes can be rejuvenated by heating. The illumination by the $\mathrm{Hg}$ lamp light $(259 \mathrm{~nm})$, successfully demonstrated to regenerate $\mathrm{K}$-Te cathodes degraded by long storage in the preparation chamber, ${ }^{5}$ does not produce any QE increase of photocathodes degraded by use in the accelerator. The results of the regeneration experiments, performed at the end of the experiments for lifetime determination, are shown in Fig. 4. The points at $26^{\circ} \mathrm{C}$ show the $\mathrm{QE}$ after degradation. The experiments started at $50{ }^{\circ} \mathrm{C}$, the cathodes were kept at this temperature for the time necessary to reach a stable $\mathrm{QE}$ value, that is reported in the figure; then the temperature was increased by steps of $30-50{ }^{\circ} \mathrm{C}$ and kept fixed at each step until a QE constant in time was measured (on the average about $20 \mathrm{~min}$ were enough to this purpose). For every cathode, the highest temperature reported in Fig. 4 is the one above which the QE no longer increases or starts to decrease. The efficiency of the regeneration process can be deduced from Table II, where the ratio between the recovered QE obtained after regeneration of the used cathodes and the initial QE measured after evaporation is reported. Both Fig. 4 and Table II do not show results concerning the SP $120{ }^{\circ} \mathrm{C}$ cathode, because its regeneration, which definitely occurs during heating, vanishes completely when this cathode cools down to room temperature.

The recovered QEs vary between $32 \%$ and $85 \%$ of the initial one, depending on the preparation recipe, whereas the temperature at which the regeneration process is completed ranges between 100 and $330{ }^{\circ} \mathrm{C}$. We have observed that all the cathodes are stable up to high temperatures; this means that even when the maximum QE during a regeneration experiment is reached at relatively low temperatures (see for example SP $90{ }^{\circ} \mathrm{C}$ and MP $120^{\circ} \mathrm{C}$ cathodes) it does not change significantly up to $300{ }^{\circ} \mathrm{C}$. Moreover, the cathodes accomplishing regeneration at high temperatures $\left(\mathrm{MP} 26^{\circ} \mathrm{C}\right.$ and MP $90^{\circ} \mathrm{C}$ ) display an increase of the QE after cooling down to room temperature (RT points in Fig. 4), which is not detected for the other cathodes.

The best rejuvenation performances are clearly to be ascribed to the SP $90{ }^{\circ} \mathrm{C}$ cathode, for which the regeneration process is completed already at $100{ }^{\circ} \mathrm{C}$ and the recovered QE is $85 \%$ of the initial $\mathrm{QE}$ value before operation. An efficient regeneration can be also obtained from a MP $26^{\circ} \mathrm{C}$ cathode, which starts to rejuvenate at $120^{\circ} \mathrm{C}$, but keeps a constant $\mathrm{QE}$ between 120 and $240{ }^{\circ} \mathrm{C}$ and needs to reach temperatures above $300{ }^{\circ} \mathrm{C}$ to accomplish the regeneration process; cooling down to room temperature of this cathode produces a $\mathrm{QE}$ increase, delivering a final QE value which is $59 \%$ of the initial one.

\section{CONCLUSIONS}

$\mathrm{K}-\mathrm{Te}$ photocathodes produced with six different recipes (MP and SP evaporated at 26, 90, and $120{ }^{\circ} \mathrm{C}$ ) have been used in an rf electron accelerator to determine their lifetime. The regeneration by heating of photocathodes degraded by use has also been investigated. It has been demonstrated that MP photocathodes have much longer lifetimes than SP photocathodes, whereas both MP and SP cathodes can be regenerated by heating. As far as lifetime and regeneration are concerned, the best performances have been displayed by a MP $26{ }^{\circ} \mathrm{C}$ cathode: its QE during operation saturates exponentially to more than $1 \%$ in about $20 \mathrm{~h}$ and can be recovered to $59 \%$ of the initial QE by heating at $320{ }^{\circ} \mathrm{C}$.

We believe the results reported in this article, which can probably be improved by further investigations, clearly demonstrate the potential of $\mathrm{K}-\mathrm{Te}$ photocathodes as a new promising electron source for photoinjectors.

\section{ACKNOWLEDGMENTS}

This research has been partially supported by the European Community HCM Contract No. ERB CHRX CT 940455 and Ministry of Economic Affairs in The Netherlands.

${ }^{1}$ C. Travier, Nucl. Instrum. Methods Phys. Res. A 340, 26 (1994).

${ }^{2}$ P. E. Oettinger, R. E. Shefer, D. L. Birx, and M. C. Green, Nucl. Instrum. Methods Phys. Res. A 272, 264 (1988)

${ }^{3}$ E. Chevallay, J. Durand, S. Hutchins, G. Suberlucq, and M. Wurgel, Nucl. Instrum. Methods Phys. Res. A 340, 146 (1994).

${ }^{4}$ S. H. Kong, J. Kinross-Wright, D. C. Nguyen, and R. L. Sheffield, Nucl. Instrum. Methods Phys. Res. A 358, 272 (1995).

${ }^{5}$ D. Bisero, B. M. van Oerle, G. J. Ernst, J. W. J. Verschuur, and W. J. Witteman, Appl. Phys. Lett. 69, 3641 (1996).

${ }^{6}$ G. J. Ernst, J. W. J. Verschuur, B. M. van Oerle, A. F. M. Bouman, J. I. M. Botman, H. L. Hagedoorn, and W. J. Witteman, Nucl. Instrum. Methods Phys. Res. A 375, 26 (1996).

${ }^{7}$ B. M. van Oerle, D. Bisero, G. J. Ernst, J. W. J. Verschuur, and W. J. Witteman, Nucl. Instrum. Methods Phys. Res. A (to be published).

${ }^{8}$ R. F. X. A. M. Mols and G. J. Ernst, Nucl. Instrum. Methods Phys. Res. A 341, 481 (1994)

${ }^{9}$ B. M. van Oerle, D. Bisero, J. W. J. Verschuur, G. J. Ernst, and W. J. Witteman, IEEE/LEOS Symposium Proceedings, Enschede, 28 November 1996 (unpublished), p. 25. 